\title{
Inserción de estudiantes de educación secundaria (ciclo diversificado) en investigaciones de bioactividad de productos naturales
}

\author{
Lanza Castillo, Vilma del V.; Henríquez Guzmán, Willian Celestino; Romero Maza, Lolymar de Los Ángeles; \\ Bennasar García, Miguel Israel \\ Inserción de estudiantes de educación secundaria (ciclo diversificado) en investigaciones de bioactividad de \\ productos naturales \\ Revista Educación, vol. 45, núm. 2, 2021 \\ Universidad de Costa Rica, Costa Rica \\ Disponible en: https://www.redalyc.org/articulo.oa?id=44066178030 \\ DOI: https://doi.org/10.15517/revedu.v45i1.43869
}

\section{(ㄷ) $\odot \Theta \Theta$}

Esta obra está bajo una Licencia Creative Commons Atribución-NoComercial-SinDerivar 3.0 Internacional. 


\section{Inserción de estudiantes de educación secundaria (ciclo diversificado) en investigaciones de bioactividad de productos naturales}

\section{Inclusion of High-School Students (Diversified Academic Tract) in Natural Plant Bioactivity Research}

Vilma del V. Lanza Castillo

Instituto Superior de Formación Docente Salomé Ureña, República Dominicana

vilma.lanza@isfodosu.edu.do

iD https://orcid.org/0000-0002-7498-9283

Willian Celestino Henríquez Guzmán

Universidad de Oriente, Venezuela

whenriquez66@gmail.com

(iD https://orcid.org/0000-0001-5254-9714

Lolymar de Los Ángeles Romero Maza

Instituto Superior de Formación Docente Salomé Ureña,

República Dominicana

lolyrome@gmail.com

(iD https://orcid.org/0000-0003-1072-6596

Miguel Israel Bennasar García

Instituto Superior de Formación Docente Salomé Ureña, República Dominicana

miguelbennasar7884@gmail.com

(iD) https://orcid.org/0000-0002-3856-0279
DOI: https://doi.org/10.15517/revedu.v45i1.43869

Redalyc: https://www.redalyc.org/articulo.oa? $\mathrm{id}=44066178030$

Recepción: 21 Octubre 2020

Aprobación: 26 Noviembre 2020

\section{Resumen:}

El involucramiento educativo en labores de investigación es trascendental para que el estudiantado se relacione con las tareas y objetivos trazados a corto y largo plazo. Se realizaron 4 proyectos de investigación (2016-2019), en los cuales se evaluaron extractos de las plantas Crescentia cujete, Lippia alba, Aloe vera, Bixa orellana, Psidium guajava y Jatropha gossypilfolia, a las que se les determinó la actividad antibacteriana y antifúngica, el efecto hipoglucemiante de extractor en ratones diabéticos, y toxicidad sobre Artemia sp. Dichas investigaciones sirvieron para optimizar el grado de motivación y el buen rendimiento del estudiantado durante el periodo de pasantías, lo cual incentivó necesidades de cooperación en la investigación. Lo anterior mejoró las relaciones entre estudiantes y el personal del laboratorio; para que las relaciones resultaran más gratificantes, se enfrentaron a las metas y objetivos personales. Se observaron otros beneficios que coadyuvaron al optimismo, sociabilidad y el compañerismo entre el estudiantado. Por otra parte, la experimentación en el área de fitoquímica favoreció el compromiso, ya que en ella se prestó atención, se cumplió con los trabajos de investigación y se logró emplazar las relaciones científico-educativas.

Palabras ClaVE: Competencias educativas, Etnobotánica, Investigación, Actividad antimicrobiana, Fitoquímica.

\section{Abstract:}

Involving students in scientific research is essential for achieving short- and long-term tasks and objectives. A series of four research projects were executed from 2016-2019, whereby extracts obtained from the Crescentia cujete, Lippia alba, Aloe vera, Bixa orellana, Psidium guajava and Jatropha gossypilfolia plants were assessed to investigate their antibacterial and antifungal properties, hypoglycemic effect on diabetic mice as well its toxicity on Artemia sp. The studies proved to be rewarding given the outstanding performance of the student interns, optimizing their motivation and leading to improved cooperation and relationships with 
colleagues and the laboratory staff. In addition to meeting their goals, other benefits were observed that contributed to optimism, sociability and fellowship among students. Through the experiments conducted with phytochemicals, students learned about commitment, gained scientific insight and developed significant scientific and educational relationships.

KEYWORDS: Educational Competencies, Ethnobotany, Investigation, Antimicrobial Activity, Phytochemistry.

\section{INTRODUCCIÓN}

Algunas personas autoras han establecido que la inserción de estudiantes en las diversas etapas de formación se basa en sus capacidades para adaptarse a la unidad educativa bajo un perfil de pasantía, a las distintas formas de socializar dentro y fuera de la institución o empresa receptora, y la manera de emprender la etapa de aprendizaje a partir de la cultura educativa (Goodenow, 1993; Fredricks, Blumenfeld y Paris, 2004; Arguedas, 2010). En este proceso de aprendizaje estudiantil, la investigación es un eje fundamental en la formación del estudiantado de la Educación Técnica Profesional (ETP). Al afrontar las diversas particularidades de enseñanza, es menester establecer una adecuada relación en el proceso aprendizaje hipotético y práctico, que destaque el perfeccionamiento en un determinado mercado ocupacional; en la ETP se distingue una etapa inicial y otra continua, como lo establece Sevilla (2017, p.7).

Se espera, mediante el proceso de pasantías, proporcionar a grupos de estudiantes, en especial para quienes experimentan dificultades económicas, una formación a nivel técnico, por medio de alternativas atractivas y pertinentes de aprendizaje, que validen los valores de la calidad y sin errores de ninguna índole que limiten la vía de cooperación entre jóvenes en ámbitos ocupacionales concretos.

Sánchez (2005), al definir las pasantías, establece que son etapas de la formación dentro de su pensum de estudio, en las cuales el estudiantado incursiona en la productividad de un país, para finalmente emprender en el argumento profesional verdadero e instaurar los espacios laborales depurados. Es frecuente que el estudiantado adopte un carácter experimental e indagatorio sobre los perfiles laborales; se entrene, superfluamente, en una posible profesión u oficio para su inserción en el mercado laboral y adquiera las herramientas básicas de un potencial empleo, que certifiquen el éxito de la evolución de una etapa escolar a una laboral. Con esta investigación se planeó el involucramiento de estudiantes de bachillerato de la Unidad Educativa Fe y Alegría San Luis de Cumaná (Sucre) en labores de investigación. Los objetivos se enfocaron en determinar el impacto educativo en el alumnado participante, que experimentó en el ambiente de los productos naturales, proceso inherente de las pasantías en el Instituto Nacional de Investigaciones Agrícolas (INIA), con sede en Cumaná (Venezuela), durante el periodo 2016-2019; además, sirvieron como requisitos parciales para obtener el título de Técnico Medio.

\section{Procedimientos metodológicos}

\section{Diseño de la investigación}

La investigación se produjo bajo la orientación cuali-cuantitativa de tipo experimental y documental. De acuerdo con lo expresado por Arias (2012):

La investigación documental es un proceso basado en la búsqueda, recuperación, análisis, critica e interpretación de datos secundarios, es decir, los obtenidos y registrados por otros investigadores en fuentes documentales: impresas, audiovisuales o electrónicas. Como en toda investigación, el propósito de este diseño es el aporte de nuevos conocimientos (p.27).

Por otra parte, la Universidad Pedagógica Experimental Libertador (2016) explica que en la investigación documental se comprueban las temáticas, a fin de enaltecer el conocimiento en diferentes ámbitos. Se basa en actividades precedentes, cuya información puede ser divulgada por la vía impresa, audiovisual o digital. 
Las revisiones documentales pasan necesariamente por la reflexión y discernimiento de los hallazgos, posturas teóricas y descripciones históricas de los aspectos relacionados con la formación a nivel técnico, en el que se ofrecen alternativas pertinentes del proceso de aprendizaje, sin distinciones que restrinjan la accesibilidad o intervención de jóvenes en determinados campos profesionales.

Con respecto a la investigación experimental, según lo señalado por Arias (2012), esta "Es un proceso que consiste en someter a un objeto o grupo de individuos, a determinadas condiciones, estímulos o tratamiento (variable independiente), para observar los efectos o reacciones que se producen (variable dependiente)" (p.34).

En el procedimiento de la evaluación investigativa, participaron grupos estudiantiles constituidos por tres (3) integrantes sin distinción de sexo, promedio académico o clase social, con edades entre 17 a 20 años, cursantes del sexto año de la Unidad Educativa Fe y Alegría San Luis, Cumaná, Sucre, Venezuela, durante los periodos académicos 2016-2019.

\section{RECOLECCIÓN E IDENTIFICACIÓN DEL MATERIAL VEGETAL}

Las muestras de las fanerógamas utilizadas fueron recolectadas en diferentes áreas verdes de Cumaná, estado Sucre, Venezuela. Se realizaron comparaciones taxonómicas con especies vegetales resguardadas en el herbario Isidro Ramón Bermúdez Romero (IRBR), del Departamento de Biología del Núcleo de Sucre, Universidad de Oriente, Venezuela.

Procesamiento y obtención de los extractos vegetales

Se efectuaron 4 investigaciones en el área de productos naturales: evaluación de la actividad biológica de Crescentia cujete L. (Tapara), en el año 2016; evaluación del efecto antibacteriano y antifúngico de Jatropha gossypiifolia L. (Euphorbiaceae) utilizada como planta medicinal en la comunidad de Caigüire, Cumaná, estado Sucre (2017); evaluación del efecto antibacteriano de Aloevera (L.) Burm. f. (Sábila) y Lippia alba (Mill) N.E.Br. ex Britton y P. Wilson (Citrona), Cumaná, municipio Sucre, estado Sucre (2018), y determinación de la actividad hipoglucemiante del onoto (Bixa orellana L.) y la guayaba (Psidium guajava L.) sobre ratones con diabetes inducida experimentalmente (2019). En este sentido, los efectos biológicos evaluados a las diferentes especies vegetales fueron orientados por el uso etnobotánico que se les ha atribuido en la región oriental de Venezuela.

Se reforzó el aprendizaje en diferentes espacios académicos y sociales, distintos al aula, con lo que se buscaba desarrollar buenas prácticas de enseñanza (Zuluaga, 1999), a fin de lograr estimular en el estudiantado la curiosidad por la experimentación y el afianzamiento, competencia que les permita vivir entre sus iguales y canalizar la reconstrucción de un patrón en el desarrollo sostenible de sociedad, tal como lo plantea Garay y Torres (2019).

Para obtener los extractos a evaluar fitoquímicamente, se emplearon diferentes órganos vegetales. Particularmente, para el procesamiento de .. cujete (tapara) se utilizaron $129 \mathrm{~g}$ del mesocarpio (pulpa), cortado en trozos, macerado en etanol $\left(48 \mathrm{~h}\right.$ ); se filtró sobre papel de filtro Whatman $\mathrm{n}^{\circ} 41$. La solución etanólica-acuosa fue concentrada a presión reducida en un rotaevaporador marca Buchi con incorporación de un baño de María $\left(36^{\circ} \mathrm{C}\right)$, con lo que se obtuvo un concentrado etanólico-acuoso (32,2 g).

Las hojas de .. guajava ( $85 \mathrm{~g}$ ) y las semillas de .. orellana $(100 \mathrm{~g})$, se trataron por separado; las semillas de .. orellana se molieron con un mortero. Ambas muestras se extrajeron en $200 \mathrm{~mL}$ de agua a ebullición durante 25 minutos; los macerados resultantes se filtraron sobre papel de filtro Whatman $\mathrm{n}^{\circ} 41 \mathrm{y}$ se secaron en una estufa $\left(40^{\circ} \mathrm{C}\right)$ hasta obtener los extractos crudos $(13,4 \mathrm{~g}$ y $7,5 \mathrm{~g}$, respectivamente).

Las hojas $(76 \mathrm{~g})$ y tallos $(23 \mathrm{~g})$ de .. alba, se secaron en la sombra a temperatura ambiente durante una semana; posteriormente, se pulverizaron separadamente, con un molino eléctrico marca Thomas. Cada órgano se maceró en alcohol isopropílico por 48 h, luego se rotaevaporaron las soluciones obtenidas de los macerados, y fueron obtenidos los extractos de los diferentes órganos vegetales. Se obtuvieron los extractos 
en alcohol isopropílico de hojas y tallos de .. alba (8 y 5,1 g, respectivamente). De la especie .. vera se extrajo el mesofilo o cristal $(64 \mathrm{~g})$, el cual se trituró con alcohol isopropílico y se filtró; se dejó secar el filtrado a $40^{\circ} \mathrm{C}$ en una estufa marca P-SeIecta para obtener el extracto isopropílico $(21,8 \mathrm{~g})$.

De .. gossypiifolia se trataron las hojas $(49 \mathrm{~g})$ y tallos $(31 \mathrm{~g})$, en ambos casos, cada muestra fue dividida en 2 partes, cuya maceración se realizó con etanol y alcohol isopropílico. Del producto de este procesamiento se obtuvieron los extractos etanólicos (5,1 g hojas y 5,5 g tallos) e isopropílicos (6,4 g hojas y 4,3 g tallos).

\section{Bioensayos}

La evaluación del efecto antibacteriano se realizó con base en la metodología descrita por Bauer, Kirby, Sherris y Turck (1966), Farjana, Zahangir, Habibur y Ekramul, (2003) y las mediciones de los halos de inhibición según Monks, Lerner, Henríquez, Farías, Schapoval, Suyenaga, Da Rocha, Schwartsmann y Mothes (2002). Se utilizaron cepas de Bacillus subtilis, Staphylococcus aureus, Enterococcus faecaiis, Proteus vulgaris, Pseudomonas aeruginosa,Escherichia coli y Klebsiella pneumoniae. En las pruebas antifúngicas se emplearon las recomendaciones de Madubunyi (1995), frente a Aspergillus niger, Rhizopus oryzae y Candida albicans. El grado de toxicidad frente al crustáceo Artemia sp. se cuantificó según el método descrito por Meyer, Ferrigni, Putnam, Jacobsen, Nichols y McLaighlin (1982). La concentración letal media $\left(\mathrm{CL}_{50}\right)$ se determinó con un programa digital planteado por Stephan (1977).

A los ratones albinos se les indujo hiperglucemia, y para determinar su efecto sobre los animales de laboratorio se siguieron las recomendaciones descritas por el CYTED (1995) y Cedeño (2019).

\section{ANÁLISIS Y DISCUSIÓN DE RESULTADOS}

El grupo de estudiantes seleccionado para los análisis con $C$. cujete determinó que el extracto etanólico del mesocarpio ejerció efecto antibacteriano contra B. subtilis $(15 \mathrm{~mm})$, lo cual coincide con lo reportado por Mahbub, Hoq, Ahmed y Sarker (2011). No se detectó efecto antifúngico ni tóxico sobre Artemia sp., con ello confirmaron que los usos etnobotánicos de especies vegetales no necesariamente se correlacionan con el hecho de subsanar varias afecciones o patologías en personas. Por otro lado, los extractos de Jatropha gossypiifolia mostraron efecto antibacteriano ante las diferentes cepas bacterianas ensayadas (Tabla 1).

TABLA 1

Efecto antibacteriano (en $\mathrm{mm}$ ) ejercido por los extractos etanólicos e isopropílicos de hojas y tallos de J. gossypiifolia.

\begin{tabular}{llllll}
\hline Microorganismos & EEH & EET & EIH & EIT & Resultado \\
Enterococcus faecalis & 20 & $17,5^{*}$ & $15^{*}$ & $11^{*}$ & $\mathrm{~S}$ \\
Klebsiella pneumoniae & $19,5^{*}$ & $18^{*}$ & $18,5^{*}$ & $10^{*}$ & $\mathrm{~S}$ \\
Escherichia coli & $20^{*}$ & $11,5^{*}$ & $14^{*}$ & $12,5^{*}$ & $\mathrm{~S}$ \\
Pseudomonas aeruginosa & 20 & $10^{*}$ & $18,5^{*}$ & $10^{*}$ & $\mathrm{~S}$ \\
Staphylococcus aureus & 19,5 & $15^{*}$ & 17,5 & $12,5^{*}$ & $\mathrm{~S}$
\end{tabular}

\footnotetext{
Fuente: Monks et al. (2002): Escala de actividad: No activo: (-); actividad débil: halo $=7-11 \mathrm{~mm}$; actividad moderada: halo $=11-16 \mathrm{~mm}$; muy avivo: halo $>16 \mathrm{~mm}$. . Leyenda: Sensible (S); EEH: extracto etanólico de hojas; EET: extracto etanólico de tallos; EU extracto en alcohol isopropílico de hojas; EIT: extracto en alcohol isopropílico de tallos. *Bacteriostático.
}

El extracto etanólico de las hojas fue el que ejerció mayor efecto antibacteriano frente a todos microorganismos probados; se tomó en consideración la escala de cuantificación propuesta por Monks et al. (2002). Ninguno de estos extractos ejerció efecto antifúngico frente a los microorganismos ensayados. En este 
sentido, al observar resultados negativos, el estudiantado los consideró un desperdicio de tiempo y esfuerzo, por ello se les exhorto a que una investigación los resultados pueden ser nulos, negativos o inesperados, como lo afirman Guimarâes, Succi, Montalli, Niederauer y Succi (2018).

La concentración plasmática de glucosa (promedio) en los animales, previo al ensayo, fue de $90 \mathrm{mg} / \mathrm{dL}$; luego de alimentarse por 30 días con una solución de sacarosa $(200 \mathrm{~g} / \mathrm{L})$ ad libitum, aumentó a 130,2 mg/dL. Posteriormente, los ratones hiperglucémicos se trataron con los extractos acuosos de .. orellana y $P$. guajava, con lo que disminuyó de manera significativa a 70 y $115 \mathrm{mg} / \mathrm{dL}$, respectivamente, con lo que se demostró la actividad hipoglucemiante de los extractos en agua de onoto y guayaba en ratones con altos niveles de glicemia inducida, cuyos resultados difieren a los presentados por Inocente, Guija, Zarzosa, Loja y Ponce (2015), quienes determinaron que el extracto a $250 \mathrm{mg} / \mathrm{kg}$ de ratón en etanol de hojas de guayaba no ocasionó efecto. Sin embargo, la dosis de $500 \mathrm{mg} / \mathrm{kg}$ causó bajas en los niveles de glicemia. El alumnado participante consideró positiva e innovadora a este tipo de actividad, por la experiencia y responsabilidad de alimentar, cambiar las camas de los ratones, clasificarlos por sexo, además de que fue la primera vez que trabajaron con animales vivos.

De manera general, se pudo apreciar que las acciones de indagación en productos naturales en el bachillerato, permitieron dominar conceptos para lograr el entendimiento en un tono impreciso, complicado y consolidado del área científico-tecnológica, a partir de saberes previos que permitieron explicar los procesos naturales. Además, el estudiantado obtuvo altos niveles de involucramiento, motivación, rendimiento y aceptación, así como una excelente participación para desarrollar trabajos en equipo, lo que incrementó la cooperación para realizar actividades que no solo fortalecen las competencias inherentes a lo cognoscitivo, sino también a lo formativo, que permitió observar una mínima apatía y ausentismo en el grupo. Se dejó en evidencia la interdisciplinariedad, y se ayudó a develar y vincular las problemáticas científicas y tecnológicas planteadas con sus posibles soluciones, enmarcadas en un contexto social-cultural particular, con las cuales se fortalecieron competencias laborales y profesionales. Todo esto coincide con lo planteado por Garay y Torres (2019), quienes expresaron, entre otras cosas, que en las investigaciones donde participa el estudiantado, este tiende a una mayor percepción conceptual e internaliza los conocimientos adquiridos al interactuar en experiencias de índole científica.

El alumnado de la Unidad Educativa Fe y Alegría experimentó y desarrolló actividades de investigación en el área de fitoquímica. Mostró un buen desenvolvimiento y motivación a la hora de realizar investigación, se observó la participación activa de todo el estudiantado en la búsqueda de información, manejo de equipos, técnicas de muestreos y ensayos, buena calidad del material presentado y defensa de las pasantías, lo cual difiere a lo propuesto por Ryan y Patrick (2001) y González (2006), quienes determinaron riesgo de consecuencias adversas en actividades extraescolares, a las que añaden malas actuaciones y, por consiguiente, inasistencia escolar; además, aseguraron que los alumnos y alumnas inexorablemente abandonan sus funciones escolares regulares.

En nuestro caso, la experiencia de aprendizaje por parte de las personas pasantes de la U.E. Fe y Alegría de la ciudad de Cumaná, evidenció buena aceptación académica por parte del estudiantado, al igual que de la directiva de dicha institución. Se logró el objetivo general en las actividades de pasantías, el cual estuvo enfocado en brindar herramientas prácticas que conllevaran a despertar interés por el área de la investigación; así como habilidades al manipular los equipos, herramientas, técnicas y asimilar un lenguaje científico, todo ello, con la finalidad de adiestrar a cada participante para el mercado de trabajo, tomó en cuenta el fundamento de la malla curricular del sistema educativo venezolano.

AI respecto, Sánchez (2005) menciona que el aprendizaje a nivel de investigación implica relevancia académica y curricular, así como para la implementación de estrategias educativas, las cuales pueden aplicarse a diferentes niveles. También enfatiza que en este tipo de prácticas existen limitaciones relacionadas con las metas objetivas, ya que estas han estado destinadas al desarrollo académico y poco relacionadas con la empleabilidad. Se deja claro que este tipo de programas que utilizan las pasantías profesionales como 
estrategias de aprendizaje promueven el fortalecimiento de los vínculos entre la escuela, la comunidad y la activación de actividades productivas futuras.

En términos generales, las pasantías se vislumbran como actividades metódicas, necesarias y de limitada permanencia, lo cual permite al alumnado irrumpir en actividades profesionales auténticas, que contrastan las hipótesis y la experiencia, lo que contribuye con las estrategias escolares y profesionales, y, de esta manera, emprende las capacidades laborales. No se observa un carácter filial, sino más bien suplementario e interdisciplinar de la pasantía dentro de la cultura laboral, la cual le otorga a esta actividad educativa un carácter obligatorio dentro del plan de formación estudiantil.

Por tal motivo, Sánchez (2005) resalta la importancia de que el estudiantado opte por favorecer la adquisición de enseñanzas novedosas relacionadas a la experiencia en el posible trabajo, ya que provoca un mayor acercamiento al mundo profesional. Dicho proceso requiere una inmersión casi total que respete la idiosincrasia de las personas pasantes y sus capacidades expresivas, que considere el entorno laboral donde confluyen sus inquietudes y necesidades; de manera que coexistan todas las partes involucradas, desde estudiantes hasta el personal directivo de la institución laboral. Se materializa una etapa evolutiva incentivada por fases de edificación, que cuenten con la anuencia de una buena práctica educativa, que es continua y de retroalimentación, lo que podría relacionarse con lo reportado por González (2006), quien indicó que el personal docente se siente animado cuando el alumnado revela exaltación, lo cual favorece a la consecución de los objetivos.

El éxito de las pasantías, tanto el desarrollo de habilidades de cada pasante o como fuente motivadora para su inserción en el campo laboral relacionado con el trabajo desempeñado durante la pasantía, dependerá de las cualidades personales destacadas por los tutores y tutoras, ya que serán una figura plena a imitar, en mejor sentido de la palabra. Dentro de los rasgos más sobresalientes a considerar se encuentra el compromiso con las labores, el cumplimiento de las tareas y puntualidad permanente al asistir al puesto de trabajo; cumplimiento con el uniforme escolar, valoración positiva de sí mismos. En términos generales, se observó una conducta activa, vigorosa, sociable y de compañerismo; del mismo modo, una participación solidaria y afectiva entre participantes, así como lo señaló Mirna Sánchez (2005)

Asimismo, se manifestó el empeño del estudiantado en cumplir con sus metas iniciales al emprender la pasantía, lo cual fue un motivo idóneo para vislumbrar su futuro de forma positiva, pues lo hace capaz de enfrentar los retos a pesar de las adversidades (Van Ryzin, Gravely, A. y Roseth 2009, p. 6; Arguedas, 2010). En este orden de ideas, Arguedas (2010) recomienda que las personas estudiantes deben esforzarse, para incentivar su espíritu de lucha y la confianza en sí mismas, a bien de lograr un involucramiento satisfactorio y significativo del estudiantado en todas las facetas del entorno educativo, para familiarizarse con el entorno laboral.

En términos generales, el estudiantado se comportó adecuadamente y demostró su potencialidad para asimilar y solventar dificultades que se suscitan en una investigación, como lo reportaron Fredricks et al. (2004). Con respecto al involucramiento cognitivo, dada la interacción entre el involucramiento y el alto rendimiento, aumentaron las expectativas del logro con respecto a cumplir con el proyecto de investigación. Es por ello que Arguedas (2010) establece que las personas involucradas cognitivamente tienen habilidad para afrontar las vicisitudes, aplicar los conocimientos adquiridos y afrontar los paradigmas relacionados con el entorno escolar.

En este sentido, Beccaria y $\mathrm{Paz}$ (2016) proponen que el periodo de pasantías debe resultar con la premisa de preparar al alumnado en el terreno laboral, como una etapa de transformación que provea apoyo ante las necesidades específicas para incursionar y adaptarse a las diferentes modificaciones en el mercado.

En el informe La red de educación técnica de Fe y Alegría: una oferta diversificada y articulada a las demandas de la población y del mercado, INAGRO (2013, p. 45), se menciona que a nivel técnico se han observado fallas, lo cual puede repercutir negativamente en el estudiantado, A pesar del prestigio en su trayectoria educativa de los instituto de formación técnica, se crea la disyuntiva en formación educativa con 
miras al desempeño profesional, con la particularidad de brindarle acceso a la población más vulnerable de la sociedad y generar una alternativa para obtener una posición digna en ella.

En la investigación realizada se obtuvieron resultados positivos al dejar a un lado las ofertas de pasantías tradicionales, y se tuvo en cuenta el desequilibrio entre los ajustes de las estrategias curriculares contextualizadas y la modernización del personal docente, atribuidos a los centros con un dogmático alcance para satisfacer y enarbolar la formación técnica en instituciones públicas al considerar las recomendaciones de Meléndez y Gómez (2008).

Cada proyecto mostró coherencia a pesar de las limitaciones epistemológicas, atribuidas posiblemente a las carencias de conocimiento en el área, restricciones monetarias y de pertenecer a una clase muy endeble de la sociedad, cuya educación técnica apunta oportunidades de capacitarse ocupacionalmente.

En los procesos de formación de jóvenes con necesidades especiales de empoderarse de la sapiencia y habilidades profesionales, habitualmente con insuficientes finanzas, se requiere implementar estrategias para subsanar los prejuicios y resquicios de quienes usan este tipo de formación educativa, al mantener el dinamismo, la sostenibilidad y sustentabilidad de la etapa de pasantías. Como se menciona en INAGRO (2013): "está claro que, sin una participación real de estos jóvenes y sus potenciales empleadores, las intervenciones en favor de su formación, tienen pocas posibilidades de éxito" (p.41). En este sentido, De León y Rodríguez (2008) mencionaron que ajustar las características y capacidades de cada persona a los requerimientos de una profesión, ayuda a alcanzar el máximo rendimiento en el trabajo, promueven la decisión de su futura carrera profesional.

\section{Conclusiones}

De las plantas probadas para determinar bioactividad, el extracto etanólico de las hojas de Jatropha gossypiifolia fue el que ejerció mayor efecto antibacteriano frente a todos los microorganismos probados. Bixa orellana fue la planta más efectiva para disminuir la concentración plasmática de glucosa en ratones hiperglucémicos. Ninguno de los extractos ensayados frente al crustáceo Artemia sp y cepas de hongos manifestaron actividad.

El estudiantado manifestó dominio de temas relacionados con los productos naturales, lo que optimizó los niveles de involucramiento, motivación, rendimiento, aceptación, participación y estímulo de trabajo en equipo. El grupo se involucró de manera cognitiva, afectiva y con sentido de responsabilidad en el cumplimiento de horarios y tareas, de esta manera logró aplicar los conocimientos adquiridos, y demostró buena disposición ante las actividades colaborativas. El alumnado pasante, al culminar sus proyectos, manifestó entusiasmo porque fue la primera vez que realizaban una investigación en el espacio de productos naturales; además, recomendó a la Unidad Educativa Fe y Alegría dar orientaciones previas a las futuras personas pasantes en INIA, para que tengan un buen desarrollo en sus proyectos de investigación.

\section{RECOMENDACIONES}

Las instituciones receptoras de pasantes deben estimular al estudiantado a participar activamente en las actividades científico-tecnológicas, a fin de garantizar su formación laboral mediante nuevas experiencias.

Se recomienda continuar con la profundización de las actividades de investigación en el área de productos naturales, para lograr el aislamiento y elucidación estructural de los compuestos responsables de la actividad biológica. 


\section{Referencias BIBLIOgráficAs}

Arguedas, I. (2010). Involucramiento de las estudiantes y los estudiantes en el proceso educativo. Revista Iberoamericana sobre Calidad, Eficacia y Cambio en Educación, 8, 63-78.

Arias, F. (2012). El proyecto de investigación. Introducción a la metodología cientifica. 6a Edición. Caracas, Venezuela: Editorial Episteme.

Bauer, A.; Kirby, W.; Sherris, I. y Turck, M. (1966). Antibiotic susceptibility testing by standarized single disk method. American journal of clinical pathology, 45(4), 493-496.

Beccaria, L. y Paz, J. (2016). La inserción precaria de mujeres y los jóvenes en el mercado laboral en América Latina y sus consecuencias para instituciones laborales. Chile: Comisión Económica para América Latina y el Caribe [CEPAL].

Cedeño, Y. (2019). Evaluación del efecto hipoglucemiante de las plantas Ziziphus mauritiana Lam (ponsigué) y Phyllantus niruri L. (buevito escondido), en ratones albinos Mus musculus con hiper-glucemia inducida experimentalmente. (Trabajo de grado). Universidad de Oriente, Departamento de Bioanálisis.

Ciencia y Tecnología para el Desarrollo (CYTED). (1995). Programa Iberoamericano de Ciencia y Tecnología para el Desarrollo. En R. Pinzón, Manual de Técnicas de Investigación. Bogotá, Colombia: Editorial R. Pinzón.

De León, T. y Rodríguez, R. (2008). El efecto de la orientación vocacional en la elección de carrera. Revista Mexicana de Orientación Educativa, 5(13), 10-16.

Farjana, N., Zahangir, A., Habibur, R. y Ekramul, H. (2003). In vitro antimicrobial activity of the compound isolated from Chloroform extract of Moringa oleifera Lam. Pakistan Journal of Biological Sciences, 6(22), 1888-1890.

Fredricks, J., Blumenfeld P. y Paris, A. (2004). School engagement: potential of the concept, state of the evidence. Review of Educational Research, 74(1), 59-109.

Garay, N. y Torres, J. (2019). Identificar las Prácticas Pedagógicas desde la Formación por Competencias en el área de Ciencias Naturales. Revista Venezolana de Sociología y Antropología, 29, 464-505.

González, M. (2006). Absentismo y Abandono Escolar: Una situación singular de la exclusión educativa. Revista Iberoamericana sobre Calidad, Eficacia y Cambio en Educación, 4(1), 1-15.

Goodenow, C. (1993). The psychological sense of school membership among adolescents: scale development and educational correlates. Psychology in the Schools, 30(1), 79-90.

Guimarâes, R., Succi, G., Montalli, V., Niederauer, A. y Succi, R. (2018). Resultados negativos en la investigación científica: aspectos éticos. Revista Bioética, 26(2), 245-250. doi: https://doi.org/10.1590/1983-804220182622 45

INAGRO. (2013). La red de educación técnica de Fe y Alegría: una oferta diversificada y articulada a las demandas de la población y del mercado (Informe Evaluación Final Proyecto). La Paz, Bolivia: Entreculturas/Fe y Alegría/ Intermón-Oxfam.

Inocente, M., Guija, E., Zarzosa, E., Loja, B. y Ponce, J. (2015). Efecto hipoglicemiante de los extractos acuoso y etanólico de Psidium guajava L. (Guayaba) en ratas diabéticas inducidas por aloxano. Horizonte Médico, 15, 41-48.

Madubunyi, I. (1995). Antimicrobial activities of the constituents of Garcinia kola seeds. International Journal of Pharmacognosy, 33(33), 232-237.

Mahbub, K., Hoq, M., Ahmed, M. y Sarker, A. (2011). In vitro antibacterial activity of Crescentia cujete and Moringa oleifera. Bangladesh Research Publications Journal, 5(4), 337-343.

Meléndez M., Sileny y Gómez V., Luis J. (2008). La planificación curricular en el aula. Un modelo de enseñanza por competencias. Laurus, 14(26), 367-392.

Meyer, B., Ferrigni, N., Putnam, J., Jacobsen, L., Nichols, D. y J. McLaighlin. (1982). Brine Shrimp: A convenient general bioassay for active plant constituents. Journal of Medicine Plant Research, 45, 31-34.

Monks, N., Lerner, C., Henriquez, A., Farias, F., Schapoval, E., Suyenaga, E., Da Rocha, A., Schwartsmann, G. y Mothes, B. (2002). Anticancer, antichemotactic and antimicrobial activities of marine sponges collected off the 
Vilma del V. Lanza Castillo, et al. Inserción de estudiantes de educación Secundaria (CiClo divers...

coast of Santa Catarina, Southern Brazil. Journal of Experimental Marine Biology and Ecology, 281(1-2), 1-12. doi: https://doi.org/10.1016/S0022-0981(02)00380-5

Ryan, A. y Patrick, H. (2001). The classroom social environment and changes in adolescents' motivation and engagement during middle school. American Educational Research Journal, 38(2), 437-460.

Sánchez, M. (2005). El aprendizaje en contextos laborales reales: el caso de las pasantías de los estudiantes universitarios. Educere, 9(30), 345-357.

Sevilla, M. P. (2017), Panorama de la educación técnica profesional en América Latina y el Caribe. Serie Políticas Sociales, No. 222 (LC/L.4287), Santiago de Chile: Comisión Económica para América Latina y el Caribe (CEPAL).

Stephan, C. (1977). Methods for calculating in LC50. En F.L. Mayer y J. Hamelink (Ed). American Society for Testing and Material (ASTM) Acuatic Toxicology and Hazard Evaluation (pp. 65-84), Pennsylvania: American Society for Testing and Materials.

Universidad Pedagógica Experimental Libertador [UPEL]. (2016). Manual de Trabajos de Grado de Especialización y Maestría y Tesis Doctorales. Vicerrectorado de Investigación y Postgrado. Sa edición. Venezuela: FEDUPEL.

Van Ryzin, M., Gravely, A. y Roseth, C. (2009). Autonomy, belongingness, and engagement in school as contributors to adolescent psychological well-being. Journal of youth and adolescence, 38(1), 1-12. doi: https://doi.org/10.1 007/s10964-007-9257-4

Zuluaga, O. (1999). Pedagogía e historia: La historicidad de la pedagogía. La enseñanza un objeto del saber. Bogotá: Editorial Universidad de Antioquia.

\section{INFORMACIÓN ADICIONAL}

Cómo citar: Lanza Castillo, V.V., Henríquez Guzmán, W.C., Romero Maza, L.A. y Bennasar García, M.I. (2021). Inserción de estudiantes de educación secundaria (ciclo diversificado) en investigaciones de bioactividad de productos naturales. Revista Educación, 45(2). Recuperado de http://doi.org/10.15517/re vedu.v45i1.43869 